\title{
Laparoscopic right hemicolectomy oriented by superior mesenteric artery for right colon cancer: efficacy evaluation with a match-controlled analysis
}

This article was published in the following Dove Press journal:

Cancer Management and Research

\author{
Weigang Dai',2,* \\ Jian Zhang ${ }^{1,2, *}$ \\ Weixin Xiong ${ }^{1,2}$ \\ Jianbo $X u^{1,2}$ \\ Shirong Cai ${ }^{1,2}$ \\ Min Tan ${ }^{1,2}$ \\ Yulong $\mathrm{He}^{1,2}$ \\ Wu Song ${ }^{1,2}$ \\ Yujie Yuan ${ }^{1,2}$ \\ 'Center of Gastrointestinal Surgery, \\ The First Affiliated Hospital, Sun \\ Yat-sen University, Guangzhou \\ 510080, Guangdong, People's Republic \\ of China; ${ }^{2}$ Center of Gastric Cancer, \\ Sun Yat-sen University, Guangzhou \\ 510080 , Guangdong, People's Republic \\ of China
}

*These authors contributed equally to this work
Correspondence: Yujie Yuan

Center of Gastrointestinal Surgery, The

First Affiliated Hospital, Sun Yat-sen

University, 58, 2nd Zhongshan Road,

Guangzhou 510080, Guangdong Province,

People's Republic of China

Tel +86 I50 I849 2852

Fax +86 2087331059

Email condor.yyj@gmail.com
Background: Laparoscopic right hemicolectomy (LRC) with a principle of D3 lymphadenectomy seems to be appropriate in treatment of right-sided colon cancer (RCC). This study aimed to evaluate clinical efficacy of superior mesenteric artery (SMA)-guided LRC (SLRC) for RCC patients.

Patients and methods: Data for RCC patients with radical resection were retrieved from our database and electronic medical records (January 2010 to December 2014). Patients undergoing SLRC procedure were compared with those undergoing conventional laparoscopic right hemicolectomy (CLRC), with a match ratio of 1:2 for group balance. Perioperative and long-term outcomes were compared between two groups.

Results: In sum, 102 matched patients were selected, with a median follow-up of 32 (range, 3-68) months. The mean operative time was significantly reduced in the SLRC group compared to the CLRC group (206.9 vs 240.0 minutes, $P=0.007$ ), with increased incidence of postoperative complications observed $(14.7 \%$ vs $8.8 \%, P=0.499)$. Average length of stay after surgery ( 7.4 vs 8.0 days), estimated blood loss ( 85.3 vs $105.4 \mathrm{~mL}$ ), number of harvested (28.4 vs 28.2) and positive (0.6 vs 0.9 ) lymph nodes, and overall costs ( $\$ 4826.9$ vs $\$ 4874.6$ ) were comparable between two groups $(P>0.05)$. The 3 -year disease-free survival rate $(89.4 \%$ vs $92.1 \%, P=0.840)$ and overall survival rate $(93.0 \%$ vs $83.1 \%, P=0.273)$ were similar in both groups. Older age ( $\geq 65$ years, $P=0.049$ ) and advanced tumor stage ( $\geq \mathrm{II}, P=0.009$ ) were independent risk factors of recurrence.

Conclusion: The perioperative and oncologic outcomes of SLRC were not superior, but comparable to CLRC. SMA-guided dissection was a feasible surgical approach in treatment of RCC. Keywords: right colon cancer, right hemicolectomy, laparoscopic surgery, D3 lymphadenectomy, outcomes

\section{Introduction}

Colorectal cancer is the third most commonly diagnosed cancer in men and the second in women, with 1,360,000 newly diagnosed patients all over the world. ${ }^{1}$ Laparoscopic surgery for colorectal cancer treatment has been dramatically improved over the last two decades ${ }^{2,3}$; however, it remains a technically required procedure for right-sided colon cancer (RCC) resections. ${ }^{4}$ The difficulty is attributed to several factors, but the major issue is vascular variability around middle colic vessels. ${ }^{1}$

To enhance the oncologic outcomes of colorectal cancer, Japanese surgeons have emphasized an essential role of D3 lymphadenectomy, based on their deep-rooted outlook that more radical extirpation of lymphatic basins would achieve more favorable oncologic results. ${ }^{5-7}$ Conventionally, D3 lymphadenectomy with medial-to-lateral 
approach was performed along the superior mesenteric vein (SMV), with surrounding lymphoid tissues dissected and central vascular ligated. However, this conventional technique almost required to open the sheath of SMV, and was thus associated with high risk of SMV injury or intraoperative conversion. ${ }^{8}$ The reported intraoperative injury to SMV was $0.2 \%-12.4 \%$, with a high conversion rate of $12.0 \%-19.9 \% .{ }^{9}{ }^{10}$

Our center proposed a modified surgical procedure for D3 lymphadenectomy: a medial-to-lateral approach and superior mesenteric artery (SMA)-navigated dissection priority to increase the number of harvested lymph nodes (LNs) in station $\mathrm{N} 3$ and to improve vessel-related complications. We herein conducted this study aiming to explore oncologic, perioperative, and treatment outcomes of SMA-guided laparoscopic right hemicolectomy (SLRC) in comparison with conventional laparoscopic right hemicolectomy (CLRC) for RCC patients.

\section{Patients and methods Patients}

This was a retrospective analysis of our colorectal cancer database at a tertiary-level teaching hospital in Southern China. Between January 2010 and December 2014, adult patients, who had confirmed diagnosis of RCC and received radical colectomy operation, were selected from the database. The selection criteria were as follows: 1) patients were 15 years of age or older, without limitation of gender; 2) patients had confirmed diagnosis of adenocarcinomas through the colonoscopy biopsy at the right colon, which included cecum, ascending colon, hepatic flexure of colon, and proximal transverse colon; 3 ) patients underwent laparoscopic surgery on scheduled time, rather than emergency operation due to severe obstruction or perforation.

The exclusion criteria were as follows: 1) patients survived $<3$ months after surgery; 2) patients had history of other malignancies or confirmed distant metastasis prior to surgery; 3) patients had $<12$ harvested LNs for tumor staging; 4) patients had previous major surgery of upper abdomen.

\section{Ethics approval and consent to participate}

The study protocol was approved by the Institutional Review Board and Human Ethics Committee of The First Affiliated Hospital of Sun Yat-sen University and carried out in accordance with the 1975 Helsinki Declaration. This was a retrospective study, so written informed content was exempted by our ethics committee. The data utilized in this study was maintained with strict confidentiality.

\section{Perioperative and oncologic outcomes}

The following parameters for comparisons of perioperative outcomes were included in this study: age, gender, body mass index (BMI), the American Society of Anesthesiologists (ASA) score, past medical history, alcohol or smoke abuse, adjuvant chemotherapy, surgical procedure, operation duration, estimated blood loss, total and positive number of LNs retrieval, length of stay (LOS) in hospital and after surgery (LOPS), postoperative morbidity. Additionally, the following variables accounting for oncologic features were compared: tumor differentiation, lymphovascular invasion, tumor size, resection margins, and tumor stage based on the American Joint Committee on Cancer guidelines (eighth edition). Of note, all harvested LNs were intentionally divided into three categories according to the Japanese Society of Colorectal Cancer guidelines: epicolic and paracolic as station N1, intermediate as station $\mathrm{N} 2$, and central nodes as station N3 (Figure S1). ${ }^{6}$

Adjuvant chemotherapy was followed for patients with pathologic stage IIB and above, and it was routinely started 3-4 weeks after surgery. Radiotherapy was not performed unless a regional recurrence occurred. Follow-up was typically every 3 months for the first year after the surgery, every 6 months for the second year, and twice a year thereafter. ${ }^{11}$ Chest and abdominopelvic computed tomography (CT) scans with enhanced contrast were performed every 6 months, with colonoscopy annually. Tumor recurrence was confirmed by radiologic or histologic methods.

\section{Study outcomes}

The primary outcome of this study was time-to-recurrence of cancer after curative resection, and the secondary outcomes included the number and distribution of dissected LNs, surgical efficiency (operation duration, intraoperative blood loss), and hospital expenditure. Of note, those who died without a reported tumor recurrence was regarded as suffering recurrence at death unless it was clearly documented otherwise. ${ }^{12}$

\section{Perioperative management}

Once admitted in our center, patients first received colonoscopy to locate tumor and confirm diagnosis, followed by contrast-enhanced CT of the chest, abdomen, and pelvis to assign a clinical stage (cTNM) through a multidisciplinary team (MDT). Magnetic resonance imaging for liver metastasis and positron emission tomography for systemic metastasis were selectively applied in specific patients. Routinely, surgical approach and systemic chemotherapy for the individual patients were determined by the MDT meeting once a week. 
The detailed surgical technique was primarily dictated by highly experienced surgeons, with open or laparoscopic fashion performed across the study period. Of note, D3 lymphadenectomy was routinely performed in our center during the study period. As previously described, open or laparoscopic D3 LN dissection must complete skeletonization and ligation of blood vessels at the site flush with their origin over the surgical trunk of the right-sided colon, followed by the en bloc removal of all connective tissues along the lateral and ventral sides of superior mesenteric vessels upward to exposure of the Henle's gastrocolic trunk, and final transection of right colic vessels and the right branch of middle colic vessels at their origins.

Five ports were employed to complete all laparoscopic procedures, with 4-6 $\mathrm{cm}$ length of midline incision made for specimen retrieval (Figure 1A). The inserted ports were located at five sites: the $10 \mathrm{~mm}$ port at $4 \mathrm{~cm}$ below the umbilicus on the midline, the $12 \mathrm{~mm}$ operative port at the intersection of the left mid-clavicular line and the midpoint perpendicular to the xiphoid umbilical line, two 5 $\mathrm{mm}$ assistant ports at the two midpoints to the right and left of the anterior superior iliac spines and the umbilicus, and an additional $5 \mathrm{~mm}$ assistant port at $3 \mathrm{~cm}$ below the costal margin on the right mid-clavicular line. Both laparoscopic approaches were started using caudal-to-cranial plus medialto-lateral method to complete en bloc resection (Figure 1B), as previously described. ${ }^{13}$

As for the SMA-guided dissection, the sheath of the SMV was not opened, with root ligation of each branched vessel performed at the end of dissection. The dissected pathway was oriented by the SMA, along its medial side until the two branches of the middle colic artery (MCA) divided at the root (Figure 1C), while the conventional dissection followed the inside space of SMV until the presence of MCA, without a full exposure of SMA needed (Figure 1D). Of note, the right branch of the MCA, or the MCA itself when there is a lack of branches, was ligated at the root along with the middle colic vein. After that, the laparoscopic dissection for both techniques was advanced in the retrocolic space, and ended at the right paracolic sulcus, in the fashion of no-touch isolation technique.

The gastroepiploic and infrapyloric LNs inside of gastrocolic ligament should be dissected once tumors were located at the hepatic flexure. Those nodes were harvested and marked as station $\mathrm{N} 2$ nodes, as mentioned earlier. In the end, a side-to-side anastomosis of the ileum and transverse colon was performed extracorporeally, and then the whole specimen was handled based on Japanese General Rules for Clinical and Pathologic Studies on Cancer of the Colon, Rectum and Anus. ${ }^{5-7}$ Importantly, the harvested LNs were mapped according to the definition of Japanese Society of Colorectal Surgeons. ${ }^{6,7}$

The efficacy of various surgical techniques was evaluated by the number of dissected LN, and the completeness of mesocolic excision, and the recurrence rate of patient groups based on long-term follow-up for at least 3 years. ${ }^{12}$ The pathologist with at least 5 years of experience was responsible for the diagnosis of preoperative and postoperative histopathology, and the determination of the final TNM stage of the resected tumor and examined LNs.

Included subjects were intentionally divided into two groups: the SLRC group, and the CLRC group. Those patients were further stratified into the following subgroups according to the highest level of metastatic LNs: N0 (negative), $\mathrm{N}^{+}, \mathrm{N}^{+}, \mathrm{N}^{+}$groups, respectively.

\section{Statistical analyses}

The clinicopathologic data of included patients were recorded prospectively and retrieved retrospectively for this study. Descriptive statistics were utilized to report demographic and oncologic characteristics. All data were stored and analyzed using the Statistical analysis Packages for Social Science software (SPSS, version 23, IBM ${ }^{\circledR}$, Chicago, IL, USA) and $\mathrm{R}$ software environment. Kaplan-Meier curves were constructed to estimate the distribution of overall survival (OS) and disease-free survival (DFS), with the log-rank test used to compare long-term outcomes between groups. As for comparing secondary outcomes, Fisher's exact test or Student's $t$-test was appropriately applied. A two-tailed $P$-value $<0.05$ was regarded as statistically significant. The propensity score matching (PSM) method was employed to reduce the possibility of selection bias and to adjust for significant differences in the baseline characteristics of enrolled cases (factors: gender, age, BMI, ASA grade, pathologic TNM stage, and tumor location).

\section{Results}

\section{Baseline characteristics}

Between January 2010 and December 2014, 145 consecutive patients with RCC met our case-selection criteria, with 74 male and 71 female adults included. Of those patients, 34 patients underwent SLRC, with the rest receiving CLRC. Comparisons of demographic variables between two groups indicated an unbalanced data distribution, especially in age $(P=0.079)$, comorbidity $(P=0.076)$, and tumor stage $(P=0.063)$; therefore, PSM at a ratio of $1: 2$ for the SLRC 

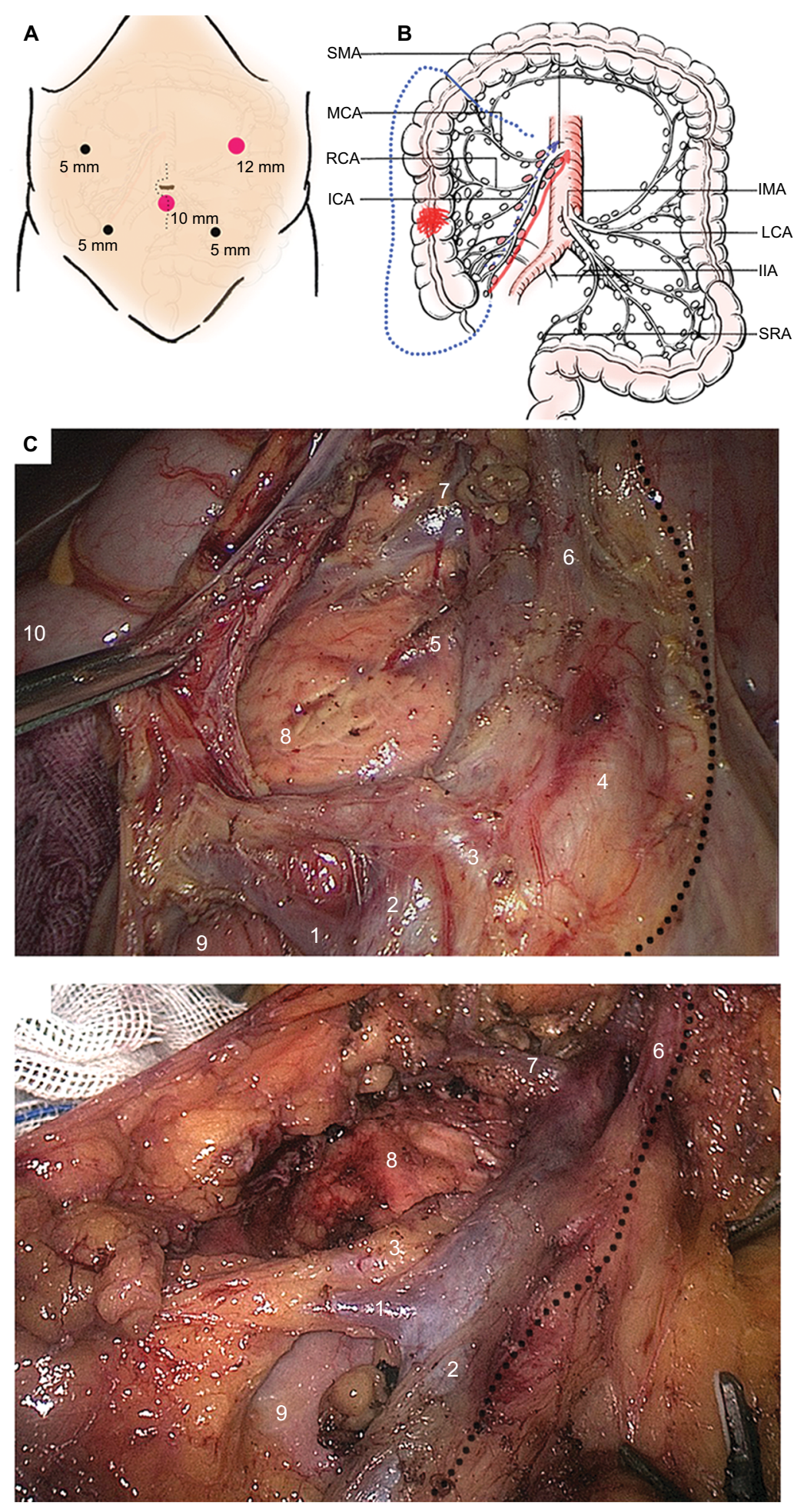

Figure I Demonstration of D3 lymphadenectomy for laparoscopic right hemicolectomy.

Notes: (A) Position of five operative ports; (B) Scope of D3 lymphadenectomy according to various surgical methods. The direction following the red arrow indicates the SLRC method, whereas that direction following the blue dotted arrow indicates the conventional SMV-guided LRC method. The blue short line indicates the cutting edge of distal bowel. (C) The typical D3 lymphadenectomy and vessel skeleton on basis of SLRC technique. LN dissection was started from the inside of SMA with a caudal-to-cranial medial approach (the dotted line). Several tributaries of SMV and SMA were ligated after sufficient space dissection (I. lleocolic vein; 2. SMV trunk or surgical trunk; 3. Root of ileocolic artery; 4. SMA trunk; 5. Superficial dorsal vein of pancreas; 6. Root of arteria colica media; 7. Henle trunk, also known as gastrocolic trunk; 8. Head of pancreas; 9. Horizontal part of duodenum; 10. Hepatic flexure). (D) The typical D3 lymphadenectomy and vessel skeleton on basis of CLRC technique. The SMA is not exposed routinely by using this technique. The dotted line stands for the inside region of lymph node dissection beyond the SMV. Those numbers indicate vessels and organs (I. Ileocolic vein; 2. SMV trunk or surgical trunk; 3. Root of ileocolic artery; 6. Root of arteria colica media; 7. Henle trunk, also known as gastrocolic trunk; 8. Head of pancreas; 9. Horizontal part of duodenum).

Abbreviations: CLRC, conventional laparoscopic right hemicolectomy; ICA, ileocolic artery; IIA, inner iliac artery; IMA, inferior mesenteric artery; LCA, left colic artery; LRC, laparoscopic right hemicolectomy; MCA, middle colic artery; RCA, right colic artery; SLRC, SMA-guided LRC; SMA, superior mesenteric artery; SMV, superior mesenteric vein; SRA, superior rectal artery. 
$(\mathrm{n}=34)$, and the matched CLRC $(\mathrm{n}=68)$ groups was applied prior to the final analysis (Table 1, Figure 2). After that, all variables including baseline laboratory results and levels of several tumor markers (Figure S2) were balanced between both groups.

Of all the 145 patients, the median follow-up period after surgery was 33 (range, 3-68) months. After the process of PSM, the median follow-up period was 32 (range, 13-68) months, which was not significantly different between two groups (median, 35vs 32 months; $P=0.431$ ). According to our selection criteria, patients who did not survive up to 3 months after surgery were excluded from this study; thus, the 30-day mortality after surgery was unavailable in this study.

\section{Perioperative outcomes analyses}

All patients received $\mathrm{R} 0$ resection without ureter, duodenum, or pancreas injury. The average LOS in hospital was markedly shorter in the SLRC group than that in the matched CLRC group ( 15.8 vs 18.2 days; $P=0.035$ ), with a similar average LOPS in both groups (7.4 vs 8.0 days; $P=0.448$ ). Additionally, the time of flatus passage $(P=0.111)$ and defecation passage $(P=0.254)$ after surgery, the incidence of postoperative morbidity $(P=0.499)$, overall costs in hospital $(P=0.686)$, and operation costs $(P=0.787)$ were not significantly different between the two groups (Table 2 ). Of note, seven $(6.9 \%)$ patients underwent unplanned laparotomy due to uncontrolled complications with conservative treatment, with four and three cases for abdominal sepsis and bowel

Table I Demographic and clinical features of included patients

\begin{tabular}{|c|c|c|c|c|c|}
\hline \multirow[t]{2}{*}{ Variable } & \multicolumn{3}{|l|}{ Overall cohort } & \multicolumn{2}{|l|}{ After PSM } \\
\hline & $\operatorname{SLRC}(n=34)$ & $\operatorname{CLRC}(n=I \mid I)$ & $P$-value & CLRC $(n=68)$ & $P$-value \\
\hline Age (years) & $62(39-84)$ & 61 (26-85) & 0.079 & $62(32-85)$ & 0.298 \\
\hline$\geq 65$ & $15(44.1)$ & $43(38.7)$ & 0.690 & $28(4 \mid .2)$ & 0.833 \\
\hline Gender (male) & $16(47.1)$ & $58(52.3)$ & 0.696 & $35(5 \mid .5)$ & 0.834 \\
\hline BMI $\left(\mathrm{kg} / \mathrm{m}^{2}\right)$ & $21.4 \pm 2.6$ & $21.9 \pm 3.1$ & 0.376 & $21.8 \pm 3.1$ & 0.467 \\
\hline Alcohol use & $15(44.1)$ & $40(36.0)$ & 0.424 & $28(4 \mid .2)$ & 0.833 \\
\hline Smoking & $9(26.5)$ & $21(18.9)$ & 0.342 & II (16.2) & 0.290 \\
\hline ASA score & & & 0.617 & & 0.857 \\
\hline I+II & $28(82.4)$ & $87(78.4)$ & & 55 (80.9) & \\
\hline III+IV & $6(17.6)$ & $24(21.6)$ & & $13(19.1)$ & \\
\hline Comorbidity & $23(67.6)$ & $54(48.6)$ & 0.076 & $51(75.0)$ & 0.484 \\
\hline Tumor location & & & 0.149 & & 0.113 \\
\hline Cecum & $6(17.6)$ & $9(8.1)$ & & $6(8.8)$ & \\
\hline Ascending colon & $21(61.8)$ & $57(51.4)$ & & $32(47.1)$ & \\
\hline Hepatic flexure & $6(17.6)$ & $39(35.1)$ & & 27 (39.7) & \\
\hline Transverse colon & I (2.9) & $6(5.4)$ & & $3(4.4)$ & \\
\hline pTNM & & & 0.063 & & 0.699 \\
\hline 0 & I (2.9) & $2(1.8)$ & & $2(2.9)$ & \\
\hline I stage & $4(11.8)$ & $10(9.0)$ & & $7(10.3)$ & \\
\hline II stage & $23(67.7)$ & $61(54.9)$ & & $39(57.4)$ & \\
\hline III stage & $6(17.7)$ & $38(34.2)$ & & $20(29.4)$ & \\
\hline Tumor grade & & & 0.952 & & 0.957 \\
\hline Well & $2(5.9)$ & $4(3.6)$ & & $3(4.4)$ & \\
\hline Moderate & $29(85.3)$ & $96(86.5)$ & & $60(88.2)$ & \\
\hline Poor & $4(I I .8)$ & $9(8.1)$ & & $4(5.9)$ & \\
\hline Undiff. & I (2.9) & $2(1.8)$ & & $\mathrm{I}(\mathrm{I} .5)$ & \\
\hline Lab. Results & & & & & \\
\hline WBC & $6.4 \pm 1.7$ & $6.6 \pm 2.1$ & 0.604 & $6.6 \pm 2.0$ & 0.486 \\
\hline $\mathrm{Hb}$ & $103.4 \pm 22.4$ & $108.6 \pm 27.2$ & 0.314 & $107.4 \pm 26.2$ & 0.442 \\
\hline PLT & $271.8 \pm 119.4$ & $292.7 \pm 100.8$ & 0.312 & $291.7 \pm 106.7$ & 0.397 \\
\hline ALB & $36.5 \pm 4.3$ & $37.6 \pm 6.0$ & 0.309 & $37.8 \pm 5.4$ & 0.230 \\
\hline TB & $8.8 \pm 4.6$ & $9.9 \pm 4.0$ & 0.216 & $10.2 \pm 3.6$ & 0.119 \\
\hline $\mathrm{Cr}$ & $74.8 \pm 17.7$ & $70.1 \pm 19.5$ & 0.206 & $69.6 \pm 17.8$ & 0.163 \\
\hline Median follow-up & $35(14-64)$ & $32(3-68)$ & 0.424 & $31(13-68)$ & 0.431 \\
\hline
\end{tabular}

Notes: Values are presented as mean \pm SD, number (\%), or median (range). Comorbidity includes hypertension, diabetes mellitus, cardiovascular disease, and other benign diseases. $P$-value vs SLCR group, with a value $<0.05$ considered as statistically significant.

Abbreviations: ALB, albumin; ASA, the American Society of Anesthesiologists; BMI, body mass index; CLRC, conventional laparoscopic right hemicolectomy; $\mathrm{Cr}$, creatine; Hb, hemoglobin; Lab., Laboratory; PLT, platelet; PTNM, pathologic TNM; PSM, propensity score matching; SLRC, superior mesenteric artery-guided laparoscopic right hemicolectomy; TB, total bilirubin; Undiff., undifferentiation; WBC, white blood cells. 


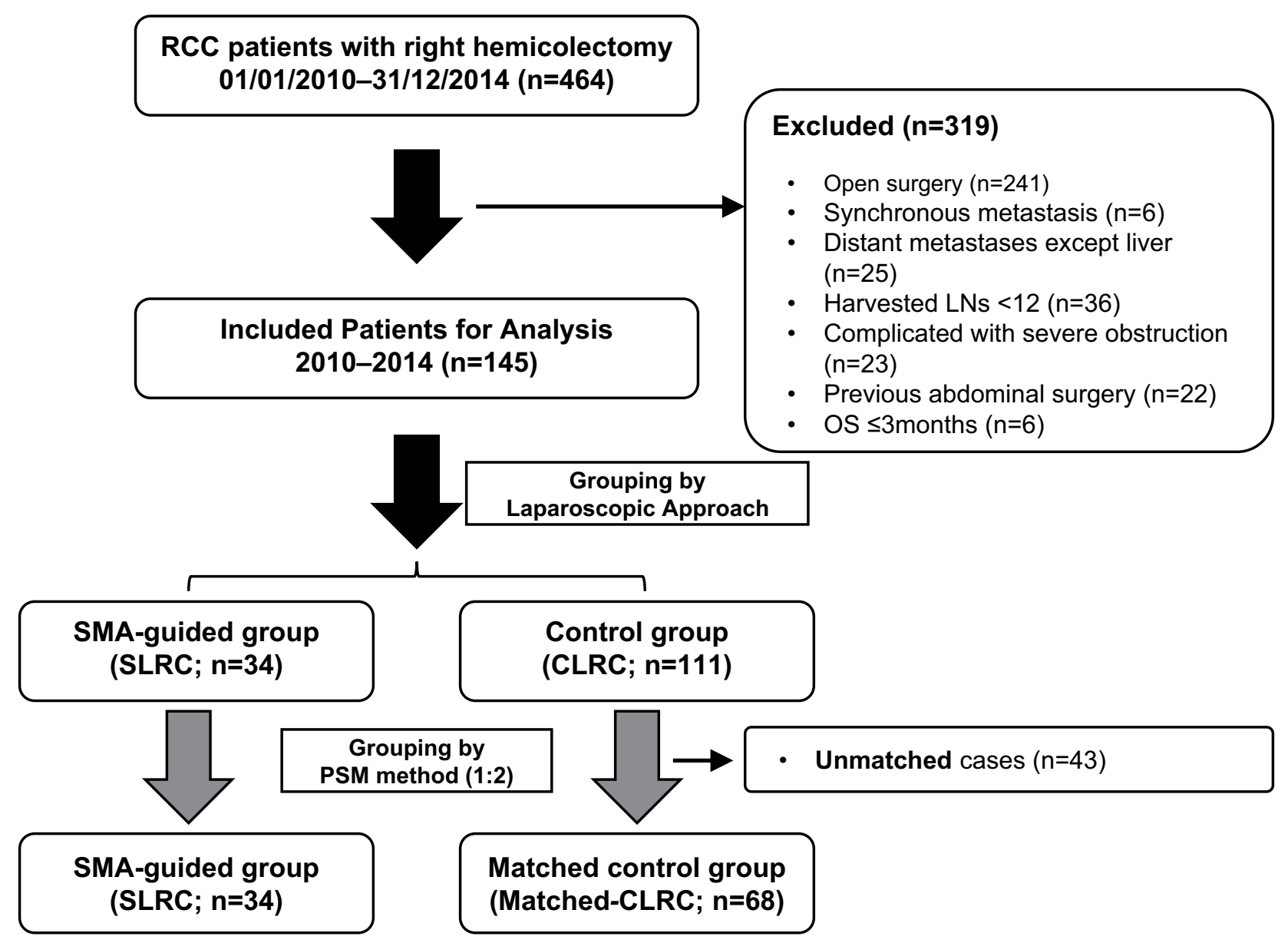

Figure 2 The flow chart of clinical data selection.

Notes: The PSM method was employed for group balance, with a ratio of I:2 determined between study and control groups. The unmatched 43 cases were removed from the comparison of long-term outcomes.

Abbreviations: CLRC, conventional laparoscopic right hemicolectomy; LNs, lymph nodes; OS, overall survival; PSM, propensity score matching; RCC, right-sided colon cancer; SLRC, SMA-guided laparoscopic right hemicolectomy; SMA, superior mesenteric artery.

obstruction, respectively. One patient suffering from anastomotic bleeding was successfully managed with endoscopic hemostasis. No patient died from such complications according to medical records.

Of the 102 matched patients, 14 cases with stage 0 and I were treated with scheduled follow-up visits alone, whereas 62 cases with stage II and 26 cases with stage III were treated with adjuvant chemotherapy, with XELOX regimen (capecitabine plus oxaliplatin) applied routinely in our center. Both tumor stage and tumor grade were comparable between the SLRC and the matched CLRC groups (Table 1).

\section{Quality evaluation of two different surgical approaches}

In the overall cohort, estimated blood loss, blood transfusion volume, number of harvested and positive LNs, and distal length of bowel resection were compared between two groups $(P>0.05$, Table 3$)$. However, operation duration (mean, 206.9 vs 237.5 minutes; $P=0.004$ ), intraoperative fluid intake (mean, 2780.8 vs $2898.4 \mathrm{~mL} ; P=0.043$ ), proximal length of bowel resection (mean, $15.4 \mathrm{vs} 18.4 \mathrm{~cm}$; $P=0.011$ ), and number of positive LNs in both N1 (mean, 0.3 vs $1.0 ; P=0.029$ ) and $\mathrm{N} 2$ (mean, 0.1 vs $0.5 ; P=0.028$ ) stations were significantly different in both groups. Of note, eleven (10.8\%) patients were converted into open laparotomy due to SMV injury $(n=6)$ and tumor progression $(n=5)$, with one case in the SLRC group and ten cases in the CLRC group. Vessel injury was not found in the SLRC group. In addition, five ( $4.9 \%$ ) patients underwent combined resection during operation, with two cases for cystectomy and three cases for adjacent intestinal resection. All the patients had a safe resection margin according to the pathologic report. By further case-matching process, patients undergoing the 
Table 2 Comparison in perioperative outcomes of right-sided colon cancer

\begin{tabular}{|c|c|c|c|c|c|}
\hline \multirow[t]{2}{*}{ Variable } & \multicolumn{3}{|l|}{ Overall cohort } & \multicolumn{2}{|l|}{ After PSM } \\
\hline & $\operatorname{SLRC}(n=34)$ & $\operatorname{CLRC}(n=I I I)$ & $P$-value & $\operatorname{CLRC}(n=68)$ & $P$-value \\
\hline LOS (day) & $15.8 \pm 5.4$ & $17.4 \pm 5.3$ & 0.117 & $18.2 \pm 5.5$ & 0.035 \\
\hline LOPS (day) & $7.4 \pm 3.1$ & $8.0 \pm 3.9$ & 0.427 & $8.0 \pm 3.7$ & 0.448 \\
\hline Flatus passage (day) & $3.8 \pm 1.5$ & $3.4 \pm 1.3$ & 0.079 & $3.4 \pm 1.2$ & 0.111 \\
\hline Defecation passage (day) & $4.3 \pm 1.7$ & $3.9 \pm 1.5$ & 0.294 & $3.9 \pm 1.5$ & 0.254 \\
\hline Complications (n) & $5(14.7)$ & $8(7.2)$ & 0.181 & $6(8.8)$ & 0.499 \\
\hline SSI & $3(8.8)$ & $5(4.5)$ & 0.335 & $4(5.98)$ & 0.580 \\
\hline Ileus or BO & $2(5.9)$ & $2(1.8)$ & 0.204 & $\mathrm{I}(\mathrm{I} .5)$ & 0.214 \\
\hline Refractory diarrhea & I (2.9) & $\mathrm{I}(0.9)$ & 0.372 & $\mathrm{I}(\mathrm{I} .5)$ & 0.614 \\
\hline Anastomotic leakage & 0 & I (0.9) & 0.579 & $\mathrm{I}(\mathrm{I} .5)$ & 0.477 \\
\hline DVT & I (2.9) & $2(1.8)$ & 0.683 & $\mathrm{I}(\mathrm{I} .5)$ & 0.614 \\
\hline Hemorrhage & $\mathrm{I}(2.9)$ & $\mathrm{I}(0.9)$ & 0.372 & 0 & 0.155 \\
\hline Overall costs $(\$)^{\mathrm{a}}$ & $8827.6 \pm 2244.4$ & $8630.2 \pm 2554.0$ & 0.686 & $8671.5 \pm 2738.8$ & 0.775 \\
\hline Operative costs (\$) & $4826.9 \pm 1200.8$ & $4801.9 \pm 1133.1$ & 0.787 & $4874.6 \pm 1 \mid 43.6$ & 0.962 \\
\hline
\end{tabular}

Notes: Values are presented as mean \pm SD or number (\%). ${ }^{a}$ Currency has been transferred from RMB into US dollar as current rate (6.4:I). Italicized P-value indicates significant differences between the two groups.

Abbreviations: BO, bowel obstruction; CLRC, conventional laparoscopic right hemicolectomy; DVT, deep venous thrombosis; LOPS, length of postoperative stay in hospital; LOS, length of stay in hospital; PSM, propensity score matching; SLRC, superior mesenteric artery-guided laparoscopic right hemicolectomy; SSI, surgical site infection.

Table 3 Quality comparison in laparoscopic right hemicolectomy between two surgical methods

\begin{tabular}{|c|c|c|c|c|c|}
\hline \multirow[t]{2}{*}{ Variable } & \multicolumn{3}{|l|}{ Overall cohort } & \multicolumn{2}{|l|}{ After PSM } \\
\hline & $\operatorname{SLRC}(n=34)$ & $\operatorname{CLRC}(n=I I I)$ & $P$-value & $\operatorname{CLRC}(n=68)$ & $P$-value \\
\hline Operation duration (minutes) & $206.9 \pm 45.4$ & $237.5 \pm 73.0$ & 0.004 & $240.0 \pm 74.8$ & 0.007 \\
\hline Estimated blood loss (mL) & $85.3 \pm 133.3$ & $117.3 \pm 104.3$ & 0.146 & $105.4 \pm 90.8$ & 0.371 \\
\hline Intraoperative blood transfusion $(\mathrm{mL})$ & $55.9 \pm 189.4$ & $47.7 \pm 136.1$ & 0.783 & $58.8 \pm 154.8$ & 0.933 \\
\hline Intraoperative fluid intake $(\mathrm{mL})$ & $2780.8 \pm 629.4$ & $2898.4 \pm 818.0$ & 0.043 & $2892.0 \pm 736.8$ & 0.454 \\
\hline Combined resection $^{a}(n)$ & I (2.9) & $4(3.6)$ & 0.666 & $2(2.9)$ & 0.743 \\
\hline Conversion (n) & I (2.9) & $10(9.0)$ & 0.242 & $6(8.8)$ & 0.406 \\
\hline Harvested LN number $(n)$ & $28.4 \pm 12.1$ & $28.7 \pm 13.5$ & 0.916 & $28.2 \pm 13.2$ & 0.935 \\
\hline NI station & $11.5 \pm 8.5$ & $11.1 \pm 6.7$ & 0.766 & $10.8 \pm 6.5$ & 0.650 \\
\hline N2 station & $6.9 \pm 4.1$ & $7.1 \pm 5.1$ & 0.868 & $6.8 \pm 5.0$ & 0.953 \\
\hline N3 station & $6.3 \pm 5.0$ & $7.9 \pm 6.0$ & 0.455 & $6.6 \pm 5.0$ & 0.658 \\
\hline Positive LN number ( $n$ ) & $0.6 \pm 1.8$ & $2.0 \pm 6.5$ & 0.203 & $0.9 \pm 2.1$ & 0.366 \\
\hline NI station & $0.3 \pm 0.8$ & $1.0 \pm 3.2$ & 0.029 & $0.5 \pm 1.6$ & 0.407 \\
\hline N2 station & $0.1 \pm 0.2$ & $0.5 \pm 2.1$ & 0.028 & $0.2 \pm 0.6$ & 0.077 \\
\hline N3 station & $0.2 \pm 0.6$ & $0.3 \pm 1.1$ & 0.401 & $0.2 \pm 0.5$ & 1.000 \\
\hline Distal length to tumor $(\mathrm{cm})$ & $14.9 \pm 6.2$ & $14.0 \pm 5.3$ & 0.389 & $13.9 \pm 5.3$ & 0.413 \\
\hline Proximal length to tumor $(\mathrm{cm})$ & $15.4 \pm 5.0$ & $18.4 \pm 6.3$ & 0.011 & $18.0 \pm 4.5$ & 0.009 \\
\hline Positive resection margin ( $\mathrm{n}$ ) & 0 & 0 & - & 0 & - \\
\hline
\end{tabular}

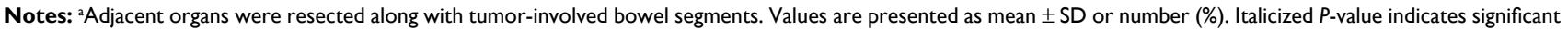
differences between the two groups. "-" indicates $P$-value not available.

Abbreviations: CLRC, conventional laparoscopic right hemicolectomy; LN, lymph node; PSM, propensity score matching; SLRC, superior mesenteric artery-guided laparoscopic right hemicolectomy.

SLRC approach still had markedly shortened operation time (mean, 206.9 vs 240.0 minutes; $P=0.007$ ) and proximal length of bowel resection (mean, $15.4 \mathrm{vs} 18.0 \mathrm{~cm} ; P=0.009$ ), as compared with those undergoing the CLRC fashion (Table 3).

As for the matched cohort, total number of dissected LNs was 2,883, with 1,430 (49.6\%), 701 (24.3\%), and 752 $(26.1 \%)$ in stations N1, N2, and N3, respectively. Meanwhile, total number of positive LNs harvested was 84 , with 49 (58.3\%), 20 (23.8\%), and 15 (17.9\%) in stations $\mathrm{N} 1, \mathrm{~N} 2$, and $\mathrm{N} 3$, respectively. As a result, the rate of $\mathrm{LN}$ metastasis was $3.4 \%$ in station $\mathrm{N} 1,2.8 \%$ in station $\mathrm{N} 2$, and $2.0 \%$ in station $\mathrm{N} 3$, with the overall metastatic rate of $2.9 \%$. Importantly, the metastatic rate in the SLRC group was significantly reduced compared to the CLRC group $(2.0 \%$ vs $3.4 \%, P=0.032$ ).

After stratifying for nodal metastasis, 77 (75.5\%) patients with negative metastasis of examined LNs were divided in N0 group, with eleven (10.8\%) cases in $\mathrm{N}^{+}$group, five $(4.9 \%)$ cases in $\mathrm{N}^{+}$group, and nine ( $\left.8.8 \%\right)$ cases in $\mathrm{N}^{+}$group. The highest level of nodal metastasis was not correlated with surgical approaches $\left(r^{2}=1.794, P=0.339\right)$. 


\section{Long-term survival outcomes}

During the follow-up periods, the 3-year cumulative OS rate was $93.0 \%$ in the SLRC group and $83.1 \%$ in the matched CLRC group, with 59.6 (95\% CI, 54.9-64.3) and 59.2 (95\% CI, 54.5-63.9) months for the estimated OS time in both groups. Importantly, the 3-year cumulative recurrence rate was $10.6 \%$ in the SLRC group and $8.9 \%$ in the matched CLRC group, with 59.3 (95\% CI, 54.3-64.4) and 60.7 (95\% CI, 57.1-64.3) months for the estimated timeto-recurrence in both groups. The differences of both OS (Figure 3) and DFS (Figure 4) were statistically insignificant by the Kaplan-Meier surviving comparison with log-rank test $(P>0.05)$.
To determine the risk factors of prognosis for such patients, a univariate cox regression analysis was performed by using 3-year OS and DFS as dependent parameters. The results indicated that older age ( $\geq 65$ years) and advanced TNM stage (stages II and III) were risk factors of poor long-term survival (Table 4). Of note, surgical approach, gender, or blood type was independent for those outcomes. By further multivariate logistical regression analysis, older age (age $\geq 65$ years, $P=0.049$ ) and advanced tumor stage (II and III, $P=0.009$ ) were independent risk factors of tumor recurrence after radical resection. However, detailed tumor location at the right-sided colon was not associated with the recurrent risk (Table 4).
A

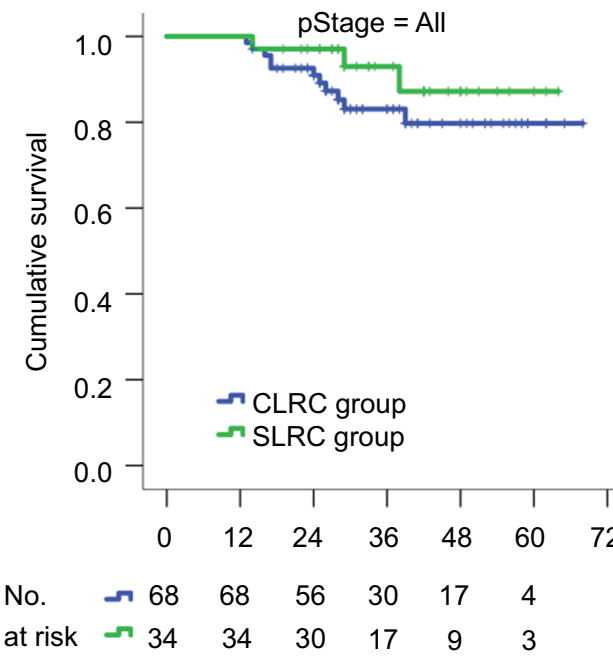

C

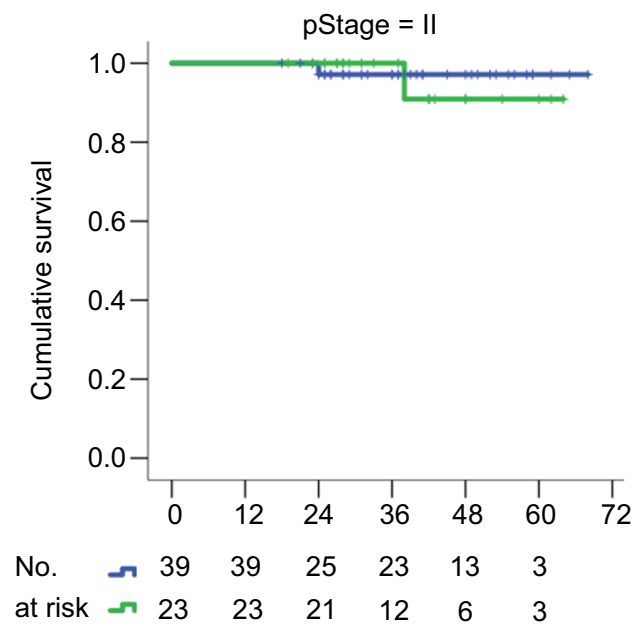

B

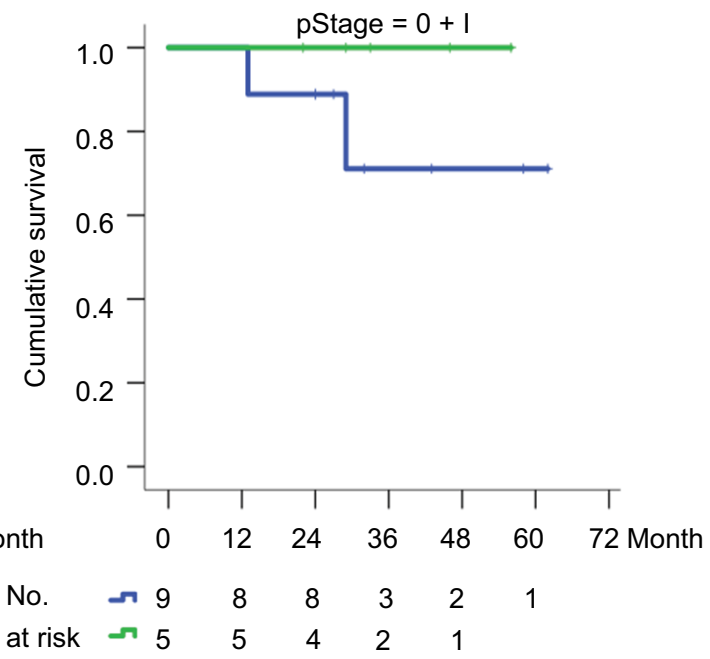

D

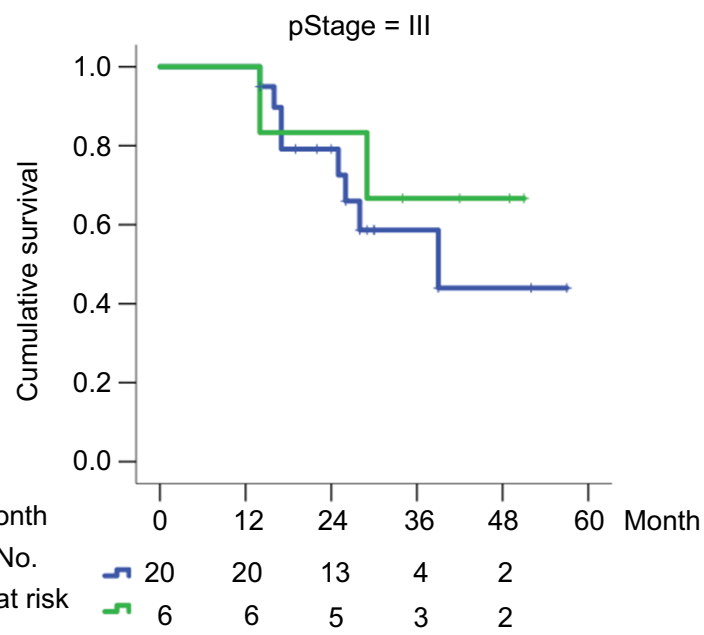

Figure 3 Kaplan-Meier curves of overall survival for RCC patients stratified by SLRC or CLRC surgical method.

Notes: (A) Any stage, $P=0.385$; (B) stages 0 and I, $P=0.246$; (C) stage II (including IIA and IIB), $P=0.705$; (D) stage III (including IIIA, IIIB, and IIIC), $P=0.504$. The 3-year rate of overall survival was compared between two groups using a log-rank test, with $P<0.05$ considered significant difference.

Abbreviations: CLRC, conventional laparoscopic right hemicolectomy; RCC, right-sided colon cancer; SLRC, superior mesenteric artery-guided laparoscopic right hemicolectomy. 
A

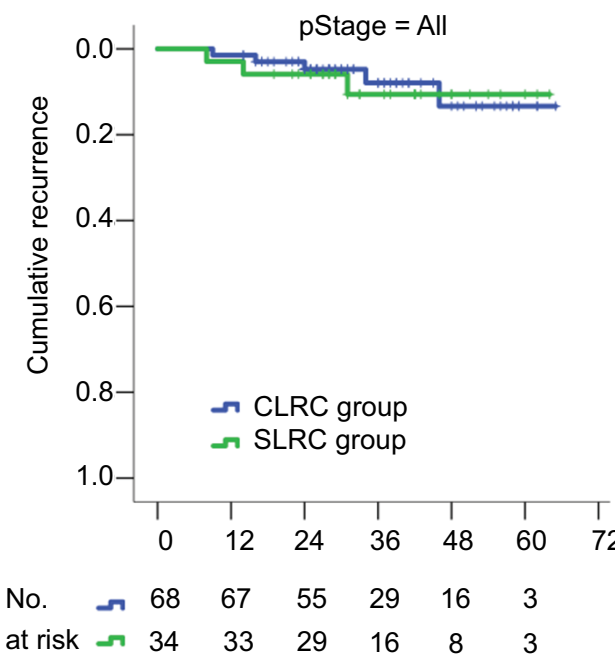

B

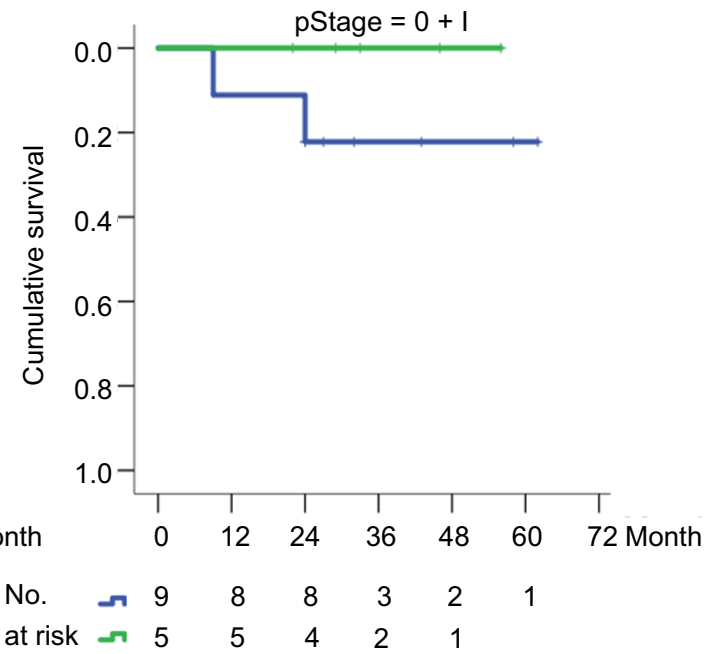

C

D
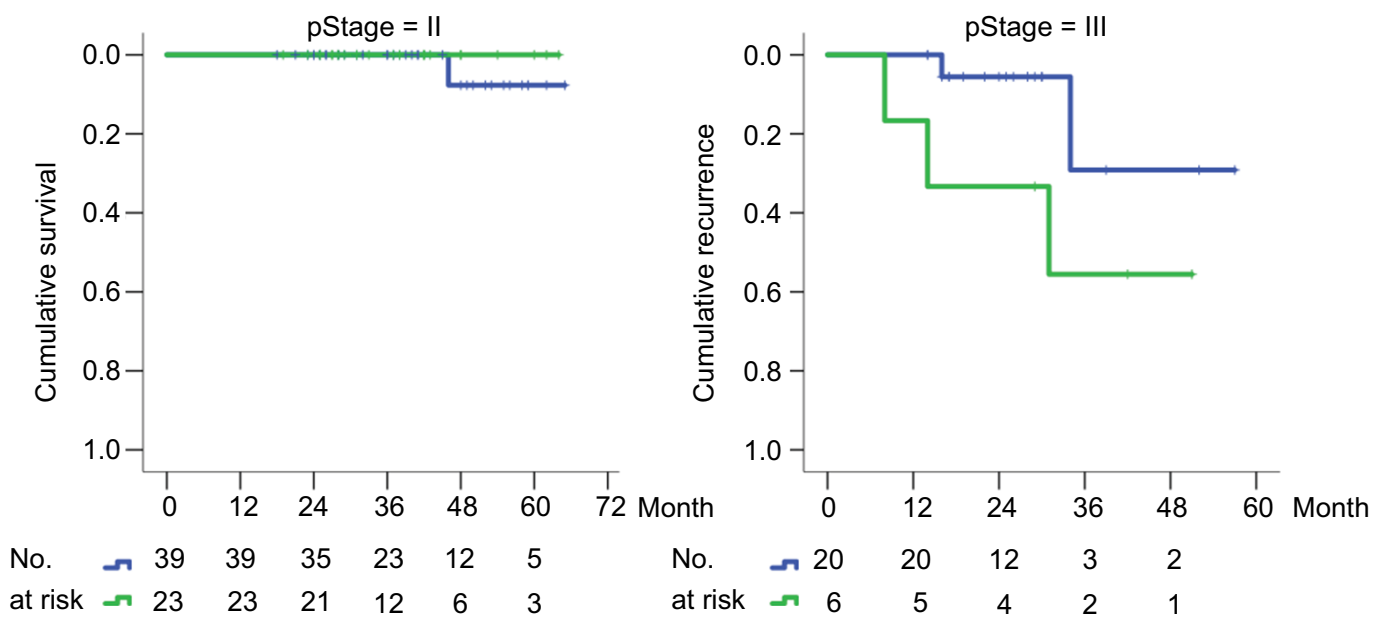

Figure 4 Kaplan-Meier curves of disease-free survival for RCC patients stratified by SLRC or CLRC surgical method.

Notes: (A) Any stage, $P=0.627$; (B) stages 0 and I, $P=0.304$; (C) stage II (including IIA and IIB), $P=0.497$; (D) stage III (including IIIA, IIIB, and IIIC), $P=0.096$. The 3-year rate of disease-free survival was compared between two groups using a log-rank test, with $P<0.05$ considered significant difference.

Abbreviations: CLRC, conventional laparoscopic right hemicolectomy; RCC, right-sided colon cancer; SLRC, superior mesenteric artery-guided laparoscopic right hemicolectomy.

\section{Discussion}

In this retrospective analysis, we evaluated the efficacy of SMA-navigated laparoscopic resection in right colon cancer. We employed laparoscopic surgery counterparts for comparison, rather than an open surgery arm, because laparoscopic resection had been shown to enhance postoperative recovery and significantly reduce the length of hospital stay. ${ }^{14,15} \mathrm{We}$ found that surgical dissection following the guidance of SMA could be successfully performed laparoscopically, with comparable specimen quality and oncologic outcomes obtained as the conventional laparoscopic dissection along the SMV. This modified approach was associated with reduced operation duration and similar length of postop- erative hospital stay compared to the conventional fashion, despite a little increased incidence of complications. Besides, it provided improved rate of SMV injury or conversion, with a comparable number of harvested LNs in station N3 obtained.

To our best of knowledge, this modified technique was rarely reported. Three main advantages might be realized from the technique. First, as the sheath of SMA was much tougher than that of SMV, the rate of vessel injury during the extension of dissection plane would be reduced without impacting on the progress of operation. Second, in theory, D3 lymphadenectomy oriented by SMA would be associated with a more extensive resection and increased number of harvested nodes around the central vessels; therefore, 
Table 4 Univariate and multivariate analyses of long-term outcomes after laparoscopic right hemicolectomy

\begin{tabular}{|c|c|c|c|c|c|c|c|c|c|}
\hline \multirow[t]{3}{*}{ Factor } & \multirow[t]{3}{*}{$N=102$} & \multirow{2}{*}{\multicolumn{3}{|c|}{$\begin{array}{l}\text { 3-year OS } \\
\text { Univariate analysis }\end{array}$}} & \multicolumn{5}{|c|}{ 3-year DFS } \\
\hline & & & & & \multicolumn{3}{|c|}{ Univariate analysis } & \multicolumn{2}{|l|}{$\begin{array}{l}\text { Multivariate } \\
\text { regression }^{\mathrm{b}}\end{array}$} \\
\hline & & Rate (\%) & OR $(95 \% \mathrm{Cl})$ & $P$-value & Rate (\%) & OR (95\% CI) & $P$-value & OR $(95 \% \mathrm{Cl})$ & $P$-value \\
\hline Age $(\geq 65:<65$ years $)$ & $43: 59$ & 64.9:94.0 & $\begin{array}{l}0.169 \\
(0.047-0.607)\end{array}$ & 0.002 & $82.2: 96.6$ & $\begin{array}{l}2.803 \\
(0.660-\mid I .90 I)\end{array}$ & 0.145 & $\begin{array}{l}0.132 \\
(0.018-0.989)\end{array}$ & 0.049 \\
\hline $\begin{array}{l}\text { BMI } \\
\left(\geq 25.0:<25.0 \mathrm{~kg} / \mathrm{m}^{2}\right)\end{array}$ & 13:89 & 83.9:87.0 & $\begin{array}{l}0.982 \\
(0.219-4.401)\end{array}$ & 0.981 & $92.3: 90.6$ & $\begin{array}{l}0.796 \\
(0.097-6.5 \mid 2)\end{array}$ & 0.831 & & \\
\hline Gender (male:female) & $51: 51$ & $83.2: 82.5$ & $\begin{array}{l}0.907 \\
(0.317-2.597)\end{array}$ & 0.856 & $89.6: 92.6$ & $\begin{array}{l}0.858 \\
(0.213-3.456)\end{array}$ & 0.830 & & \\
\hline $\begin{array}{l}\text { Blood type } \\
\text { (A:AB:B:O) }\end{array}$ & $22: 4: 29: 47$ & $\begin{array}{l}\text { 87.2:37.5: } \\
85.4: 90.6\end{array}$ & $\begin{array}{l}1.045 \\
(0.191-9.112)\end{array}$ & 0.078 & $\begin{array}{l}\text { 90.7:100: } \\
\text { 89.7:91.8 }\end{array}$ & $\begin{array}{l}1.068 \\
(0.195-5.866)\end{array}$ & 0.956 & & \\
\hline $\begin{array}{l}\text { Tumor location } \\
\text { (cecum:ascending: } \\
\text { hepatic:transverse) }\end{array}$ & 12:53:33:4 & $\begin{array}{l}90.0: 85.2: \\
90.3: 75.0\end{array}$ & $\begin{array}{l}\text { I.109 } \\
(0.587-2.092)\end{array}$ & 0.752 & $\begin{array}{l}\text { 100:87.9: } \\
93.8: 75.0\end{array}$ & $\begin{array}{l}1.674 \\
(0.798-3.51 \mathrm{I})\end{array}$ & 0.168 & $\begin{array}{l}0.060 \\
(0.003-1.393)\end{array}$ & 0.079 \\
\hline $\begin{array}{l}\text { Surgical approach } \\
\text { (SLRC:CLRC) }\end{array}$ & $34: 68$ & $93.0: 83.1$ & $\begin{array}{l}0.489 \\
(0.136-1.756)\end{array}$ & 0.273 & $89.4: 92.1$ & $\begin{array}{l}1.159 \\
(0.277-4.851)\end{array}$ & 0.840 & & \\
\hline TNM stage (0+I:II:III) & $14: 62: 26$ & $\begin{array}{l}82.5: 94.9: \\
60.3\end{array}$ & $\begin{array}{l}4.581 \\
(1.696-12.378)\end{array}$ & 0.003 & $\begin{array}{l}\text { 92.9:94.7: } \\
62.9\end{array}$ & $\begin{array}{l}0.053 \\
(0.006-0.466)\end{array}$ & 0.002 & $\begin{array}{l}0.013 \\
(0.001-0.219)\end{array}$ & 0.009 \\
\hline $\begin{array}{l}\text { Complications }{ }^{\mathrm{a}} \\
\text { (Yes:No) }\end{array}$ & 11:91 & $90.0: 86.0$ & $\begin{array}{l}1.890 \\
(0.246-14.495)\end{array}$ & 0.540 & $90.9: 91.0$ & $\begin{array}{l}0.430 \\
(0.086-2.153)\end{array}$ & 0.305 & & \\
\hline
\end{tabular}

Notes: any postoperative morbidity was considered as complications and pooled together for survival comparison. ${ }^{\mathrm{b}}$ Those factors were qualified from the univariate analysis of disease-free survival, whose $P$-values were $<0.20$.

Abbreviations: BMI, body mass index; CLRC, conventional laparoscopic right hemicolectomy; DFS, disease-free survival; OS, overall survival; SLRC, superior mesenteric artery-guided laparoscopic right hemicolectomy.

the chance of residual central nodal metastasis would be minimized compared to dissection oriented by SMV. In our results, although the number of examined LNs in station N3 (mean, 6.3 vs $6.6, P=0.658$ ) was similar in both surgical approaches, the metastatic rate $(2.0 \%$ vs $3.4 \%, P=0.032)$ was significantly reduced. The combining effect of increased number of harvested LNs and reduced number of positive LNs, limited sample size, and heterogenous nodal retrieval from specimen might be attributed to such findings. At last, the exposure of SMA was much easier in obese patients than SMV due to enlarged movement of pulsating artery, which also enhanced the safety and promptness of D3 lymphadenectomy during surgery. In our study, the mean operation time was significantly reduced by 30 minutes using SMA-guided surgical dissection compared to conventional technique, which was also comparable to reported results from similar studies. ${ }^{12,13}$

The great concern about artery-oriented dissection was the risk of injury to autonomic nerves and accompanied bowel dysfunction, such as refractory constipation, massive diarrhea, and postoperative ileus. In our center, we performed a higher level of dissection at least $5 \mathrm{~mm}$ inside to the trunk of SMA, which might reduce unnecessary damage to nervous plexus. According to our postoperative outcomes, the incidence of diarrhea, constipation, or ileus was not more than $6 \%$, which was comparable to the conventional SMV-guided dissection technique. In addition, superior mesenteric vessel injury during right colectomy is a potentially devastating iatrogenic injury with catastrophic implications. ${ }^{10,16}$ Preoperative roundup of anatomical vascular variations along with precise exposure of superior mesenteric vessels could be essential to the prevention of such injury during surgery. Besides, early intraoperative consultant on an experienced vascular surgeon, adequate anticoagulation, and a low threshold for second-look laparotomy might be valuable to obtain a more favorable outcome.

To improve oncologic long-term outcomes of colon cancer, D3 lymphadenectomy was suggested by Japanese surgeons; meanwhile, complete mesocolic excision (known as CME), which constitutes sufficient resection of mesocolon along the embryonic dissection plane with central vascular ligation, was emphasized by European surgeons. ${ }^{17-19}$ Numerous studies have confirmed that both techniques were qualified for achieving sufficient resection margin and central LNs dissection. ${ }^{13,20-22}$ It has been well recognized that dissection along the embryonic converging plane is associated with less bleeding and injury to adjacent organs, and better oncologic outcomes. ${ }^{23}$ In our center, we did not differentiate those surgical principles when resecting the tumor. However, the qualities of operation and specimen were qualified to obey both rules. The average length of resected bowel around primary tumor was more than $20 \mathrm{~cm}$ in the current study; besides, only $7.7 \%$ 
of 464 RCC patients (Figure 2) had an insufficient number of examined LNs ( $<12$ nodes) and were excluded from the final analysis to reduce the shift of tumor staging.

Our study had some shortcomings. First, although the PSM method had been applied, selection bias and several confounding factors would be inevitable due to the retrospective nature. Second, the sample size for the SLRC group was relatively small, as compared to the controlled group; thus, the current statistical significance might be underestimated, especially for the number of harvested and metastatic LNs in station N3. More cases collected prospectively would be helpful to obtain more reliable results. Third, patients with advanced tumor stage, especially for stage III, would prefer open surgery in China, and they were few included for this study. At last, the role of adjuvant chemotherapy was not explored, without detailed information of oncogene mutations available for the current cohort. Our long-term results for RCC patients required to validate with further longer follow-up period and other clinical trials with similar study design.

\section{Conclusion}

In summary, the current analysis with a matched cohort suggested that SLRC under the principle of D3 lymphadenectomy was a safe and feasible surgical approach for the management of right colon cancer. The perioperative and long-term outcomes by using this technique were comparable to the conventional laparoscopic method, although the incidence of postoperative complications was a little increased. With a limited follow-up time and small sample size, welldesigned clinical trials with multicenter collaboration are necessary to validate the results of this study.

\section{Acknowledgments}

This study was supported by grants from National Natural Science Foundation of China (81401307 and 81472260) and Medical Science, Technology Research Fund Project in GuangDong province (A2015112, 2015A030313053, and 2015A030313053), and Fundamental Research Funds for the Central Universities (15ykpy17). Donglian Chen, Ze-e $\mathrm{Xu}$, and Lifeng Lu must be thanked for their dedicated work in telephone follow-up and dataset management.

\section{Author contributions}

All authors contributed to data analysis, drafting and revising the article, gave final approval of the version to be published, and agree to be accountable for all aspects of the work.

\section{Disclosure}

The authors report no conflicts of interest in this work.

\section{References}

1. Kwak HD, Ju JK, Lee SY, Kim CH, Kim YJ, Kim HR. A comparison of laparoscopic and open D3 lymphadenectomy for transverse colon cancer. Int J Colorectal Dis. 2017;32(12):1733-1739.

2. Abraham NS, Young JM, Solomon MJ. Meta-analysis of short-term outcomes after laparoscopic resection for colorectal cancer. Br J Surg. 2004;91(9):1111-1124.

3. Ng SS, Leung KL, Lee JF, Yiu RY, Li JC, Hon SS. Long-term morbidity and oncologic outcomes of laparoscopic-assisted anterior resection for upper rectal cancer: ten-year results of a prospective, randomized trial. Dis Colon Rectum. 2009;52(4):558-566.

4. Hamabe A, Park S, Morita S, et al. Analysis of the vascular interrelationships among the first jejunal vein, the superior mesenteric artery, and the middle colic artery. Ann Surg Oncol. 2018;25(6):1661-1667.

5. Hashiguchi Y, Hase K, Ueno H, Mochizuki H, Shinto E, Yamamoto J. Optimal margins and lymphadenectomy in colonic cancer surgery. Br J Surg. 2011;98(8):1171-1178.

6. Watanabe T, Itabashi M, Shimada Y, et al. Japanese Society for Cancer of the Colon and Rectum (JSCCR) guidelines 2010 for the treatment of colorectal cancer. Int J Clin Oncol. 2012;17(1):1-29.

7. Watanabe T, Muro K, Ajioka Y, et al. Japanese Society for Cancer of the Colon and Rectum (JSCCR) guidelines 2016 for the treatment of colorectal cancer. Int J Clin Oncol. 2018;23(1):1-34.

8. Feng B, Ling TL, Lu AG, et al. Completely medial versus hybrid medial approach for laparoscopic complete mesocolic excision in right hemicolon cancer. Surg Endosc. 2014;28(2):477-483.

9. Bertelsen CA, Neuenschwander AU, Jansen JE, et al. Short-term outcomes after complete mesocolic excision compared with 'conventional' colonic cancer surgery. Br J Surg. 2016;103(5):581-589.

10. Freund MR, Goldin I, Reissman P. Superior mesenteric vein injury during oncologic right colectomy: current vascular repair modalities. Vasc Endovascular Surg. 2018;52(1):11-15.

11. Ren Y, Ye J, Wang Y, et al. The optimal application of transrectal ultrasound in staging of rectal cancer following neoadjuvant therapy: a pragmatic study for accuracy investigation. J Cancer. 2018;9(5):784-791.

12. Liang JT, Lai HS, Huang J, Sun CT. Long-term oncologic results of laparoscopic D3 lymphadenectomy with complete mesocolic excision for right-sided colon cancer with clinically positive lymph nodes. Surg Endosc. 2015;29(8):2394-2401.

13. Wang Y, Zhang C, Zhang D, Fu Z, Sun Y. Clinical outcome of laparoscopic complete mesocolic excision in the treatment of right colon cancer. World J Surg Oncol. 2017;15(1):174.

14. van der Pas MH, Haglind E, Cuesta MA, et al. Laparoscopic versus open surgery for rectal cancer (COLOR II): short-term outcomes of a randomised, phase 3 trial. Lancet Oncol. 2013;14(3):210-218.

15. Kennedy RH, Francis EA, Wharton R, et al. Multicenter randomized controlled trial of conventional versus laparoscopic surgery for colorectal cancer within an enhanced recovery programme: EnROL. $J$ Clin Oncol. 2014;32(17):1804-1811.

16. Freund MR, Edden Y, Reissman P, Dagan A. Iatrogenic superior mesenteric vein injury: the perils of high ligation. Int $J$ Colorectal Dis. 2016;31(9):1649-1651.

17. Hohenberger W, Weber K, Matzel K, Papadopoulos T, Merkel S. Standardized surgery for colonic cancer: complete mesocolic excision and central ligation--technical notes and outcome. Colorectal Dis. 2009;11(4):354-364; discussion 364-365.

18. Hogan AM, Winter DC. Mesocolic plane surgery: just plain surgery? Colorectal Dis. 2009;11(4):430-431.

19. West NP, Hohenberger W, Finan PJ, Quirke P. Mesocolic plane surgery: an old but forgotten technique? Colorectal Dis. 2009;11(9):88-989. 
20. West NP, Hohenberger W, Weber K, Perrakis A, Finan PJ, Quirke P. Complete mesocolic excision with central vascular ligation produces an oncologically superior specimen compared with standard surgery for carcinoma of the colon. J Clin Oncol. 2010;28(2):272-278.

21. West NP, Kobayashi H, Takahashi K, et al. Understanding optimal colonic cancer surgery: comparison of Japanese D3 resection and European complete mesocolic excision with central vascular ligation. J Clin Oncol. 2012;30(15):1763-1769.
22. Kobayashi H, West NP, Takahashi K, et al. Quality of surgery for stage III colon cancer: comparison between England, Germany, and Japan. Ann Surg Oncol. 2014;21(Suppl 3):S398-S404.

23. Bokey EL, Chapuis PH, Dent OF, Mander BJ, Bissett IP, Newland RC. Surgical technique and survival in patients having a curative resection for colon cancer. Dis Colon Rectum. 2003;46(7):860-866. 


\section{Supplementary materials}
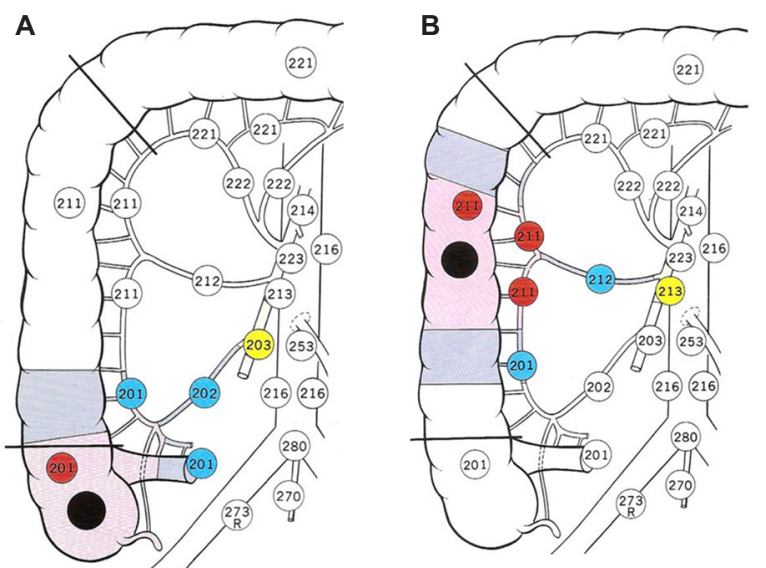

C

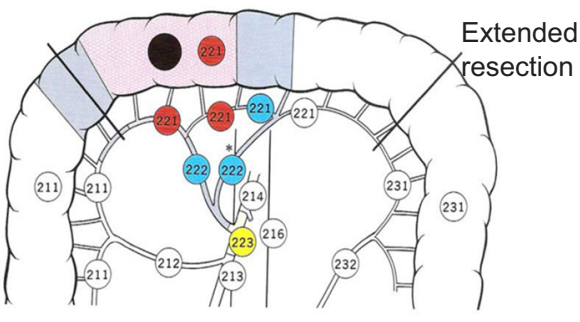

N1 Lymph node

N2 Lymph node

N3 Lymph node

Figure SI Lymph node mapping along the feeding artery and colonic wall for right-sided colon cancer.

Notes: (A) Cecal cancer, (B) ascending colon cancer, and (C) transverse colon cancer. Copyright @2012. Japanese Society of Clinical Oncology. Reproduced from Watanabe T, Itabashi M, Shimada Y, et al. Japanese Society for Cancer of the Colon and Rectum (JSCCR) guidelines 2010 for the treatment of colorectal cancer. Int J Clin Oncol. 20I2; I7(I): I-29.

A

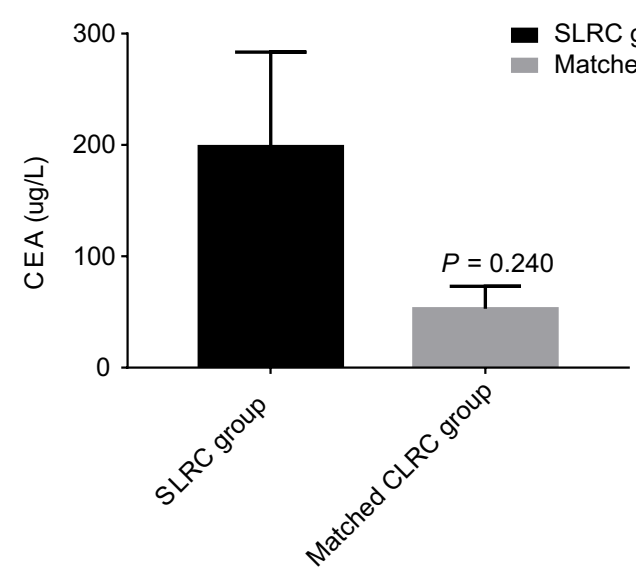

C
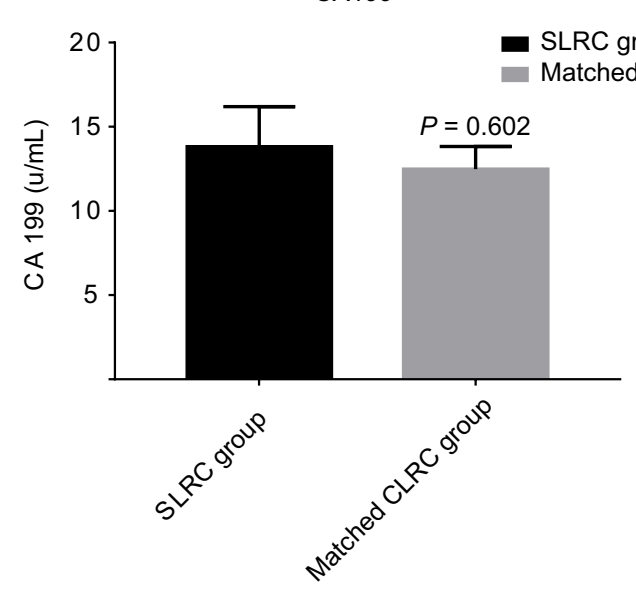

D

CA125

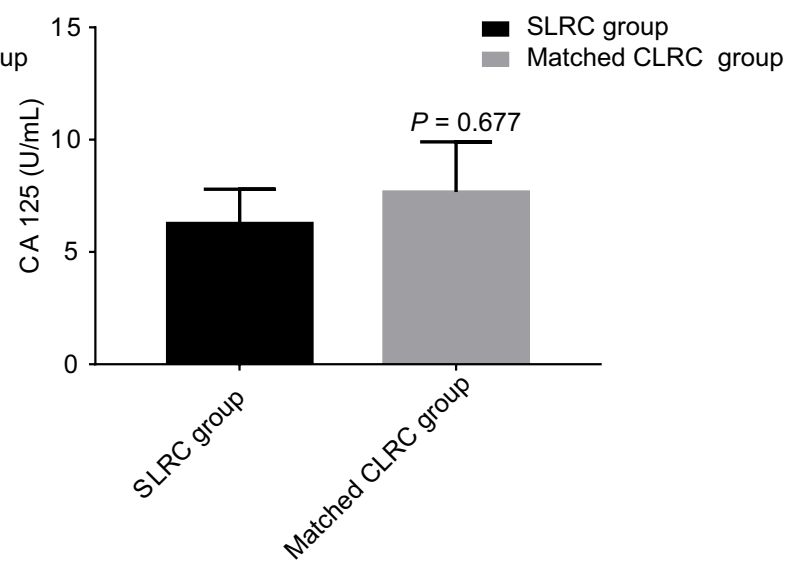

D

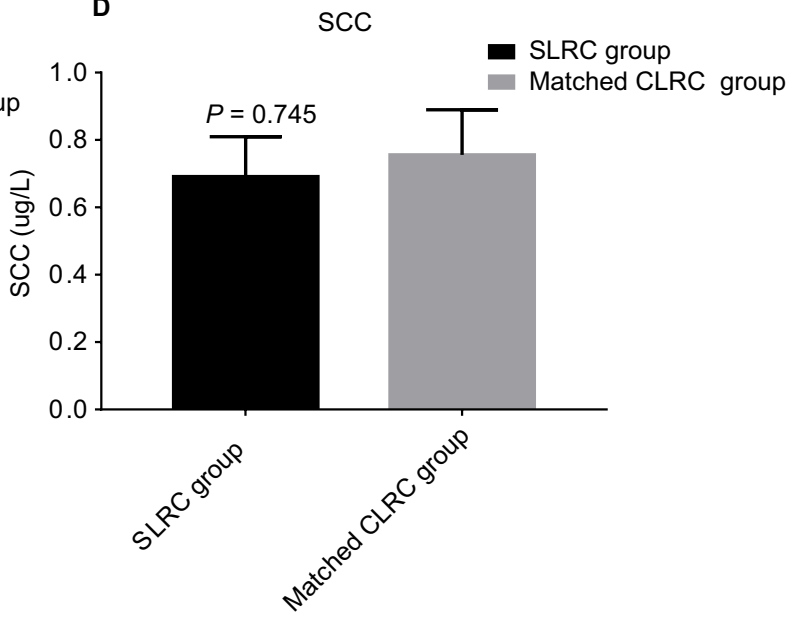

Figure $\mathbf{S 2}$ The serum baseline levels of common tumor markers prior to surgery.

Notes: (A) Serum levels of CEA, (B) serum levels of CA 125, (C) serum levels of CA 199, and (D) serum levels of squamous cell carcinoma.

Abbreviations: CA, carbohydrate antigen; CEA, carcinoembryonic antigen; CLRC, conventional laparoscopic right hemicolectomy; SCC, squamous cell carcinoma; SLRC, superior mesenteric artery-guided laparoscopic right hemicolectomy. 


\section{Reference}

1. Watanabe T, Itabashi M, Shimada Y, et al. Japanese Society for Cancer of the Colon and Rectum (JSCCR) guidelines 2010 for the treatment of colorectal cancer. Int J Clin Oncol. 2012;17(1):1-29.

\section{Publish your work in this journal}

Cancer Management and Research is an international, peer-reviewed open access journal focusing on cancer research and the optimal use of preventative and integrated treatment interventions to achieve improved outcomes, enhanced survival and quality of life for the cancer patient. The manuscript management system is completely online and includes

a very quick and fair peer-review system, which is all easy to use. Visit http://www.dovepress.com/testimonials.php to read real quotes from published authors. 